\title{
Disentangling stellar activity and planetary signals
}

\section{Isabelle Boisse ${ }^{1,2}$, François Bouchy ${ }^{1,3}$, Guillaume Hébrard ${ }^{1,3}$, Xavier Bonfils ${ }^{4,5}$, Nuno Santos ${ }^{2}$ and Sylvie Vauclair ${ }^{6}$}

\author{
${ }^{1}$ Institut d'Astrophysique de Paris, Université Pierre et Marie Curie, UMR7095 CNRS, \\ 98bis bd. Arago, 75014 Paris, France, email: iboisse@iap.fr \\ ${ }^{2}$ Centro de Astrofísica, Universidade do Porto, Rua das Estrelas, 4150-762 Porto, Portugal \\ ${ }^{3}$ Observatoire de Haute Provence, CNRS/OAMP, 04870 St Michel l'Observatoire, France \\ ${ }^{4}$ Laboratoire d'Astrophysique de Grenoble, Observatoire de Grenoble, Université Joseph \\ Fourier, CNRS, UMR 5571, 38041, Grenoble Cedex 09, France \\ ${ }^{5}$ Obs. de Genève, Université de Genève, 51 Ch. des Maillettes, 1290 Sauverny, Switzerland \\ ${ }^{6}$ LATT-UMR 5572, CNRS \& Université P. Sabatier, 14 Av. E. Belin, 31400 Toulouse, France
}

\begin{abstract}
High-precision radial-velocimetry (RV) is until now the more efficient way to discover planetary systems. Moreover, photometric transit search missions like CoRoT and Kepler, need spectroscopic RV measurements to establish the planetary nature of a transit candidate and to measure the true mass. An active star has on its photosphere dark spots and bright plages rotating with the star. These inhomogeneities of the stellar surface can induce a variation of the measurement of the RV, due to changes in lines shapes and not to a Doppler motion of the star (e.g. Queloz et al. 2001; Desort et al. 2007; Boisse et al. 2009). We study how the Keplerian fit used to search for planets in RV data is confused by spots and we test an approach to subtract RV jitter based on harmonic decomposition of the star rotation. We use simulations of spectroscopic measurements of rotating spotted stars and validate our approach on active stars monitored by high-precision spectrograph HARPS: CoRoT-7 and $\iota$ Hor.
\end{abstract}

Keywords. techniques: radial velocities, planetary systems, stars: activity, stars: individual (CoRoT-7, ८ Hor)

\section{Dark spot simulations}

SOAP is a tool that calculates the photometric, RV and line shape modulations induced by one (or more) cool spots on a rotating stellar surface. The RV modulations due to a spot as a function of time for different inclinations $i$ of the star with the line of sight and different spot latitudes lat. These two parameters clearly modify the pattern of the RV modulation. Main peaks are clearly detected in the Lomb-Scargle periodograms at the rotational period of the star $\mathrm{P}_{\text {rot }}$, as well as the two-first harmonics $\mathrm{P}_{\text {rot }} / 2$ and $\mathrm{P}_{\text {rot }} / 3$. The periods detected in the periodograms are the same for: 1 ) stars with different inclinations, 2) spots at different latitudes, 3) spot size and/or temperature varying with time, 4) several spots on the stellar surface.

The purpose is to remove or at least to reduce the stellar activity signals in order to identify a planetary signal hidden in the RV jitter. The Lomb-Scargle periodogram corresponds to sinusoidal decompositions of the data. Three sinusoids with periods fxed at the rotational period $\mathrm{P}_{\text {rot }}$, and its two-first harmonics $\mathrm{P}_{\text {rot }} / 2$ and $\mathrm{P}_{\text {rot }} / 3$ reduce the semi- amplitude of the RV jitter by more than $87 \%$.

\section{Application to real data}

CoRoT-7. Queloz et al. (2009) (Q09) reported the intensive campaign carried out with HARPS. RV variations are dominated by the activity of the star. Using our harmonic 

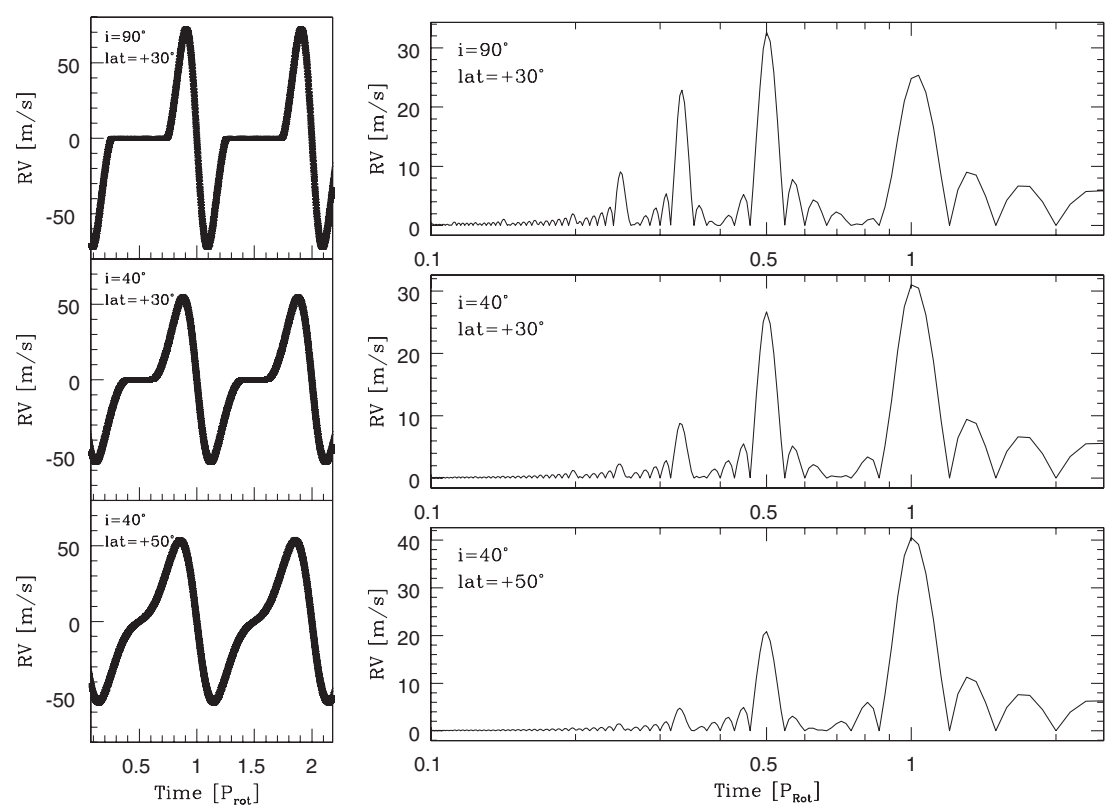

Figure 1. Left: RV modulations due to one spot as a function of time (expressed in rotational period unit). At $t=0$, the dark spot of $1 \%$ of the visible stellar surface is in front of the line of sight. The shape of the signal changes with the inclination $i$ of the star and the latitude lat of the spot, labelled in the top left of each panel. Right: Lomb-Scargle periodograms of the three RV modulations showed at the left. The fundamental frequency, $P_{r o t}$, and its first harmonics are detected.

filter, we detected in these data the two planets. Then, we fitted simultaneously the stellar activity and planetary system, instead of the method used by Q09 and obtain new values for the masses. We estimate approximately that a systematic noise due to active jitter of $1.5 \mathrm{~ms}^{-1}$ must be added quadratically to the error bars. We then find for the masses $5.7 \pm 2.5 \mathrm{M}_{\text {Earth }}$ for CoRoT-7b agreeing with the value of Q09 and 13.2 \pm 4.1 $\mathrm{M}_{\text {Earth }}$, slightly higher than the published value, for CoRoT-7c.

$\iota$ Hor. Iota Hor is a young G0V star with a long-period exoplanet. We studied 8 nights of HARPS data (Vauclair et al. 2008) showing an explicit RV scatter of about $15 \mathrm{~m} / \mathrm{s}$ probably due to stellar activity. We want to characterize the active jitter and to search for a possible hidden Doppler motion. Based on simulation studies, we fitted 3 sinusoids with period fixed at $\mathrm{P}_{\text {rot }}, \mathrm{P}_{\text {rot }} / 2$ and $\mathrm{P}_{\text {rot }} / 3$.

We do not detect in the data a short-period companion. Nevertheless, we would like to know if we have subtracted the RV shift due to a companion subtracting the effect of activity. We ran simulations adding RV due to fake planets to the data. We excluded the presence of planet with minimum mass between 6 and $10 \mathrm{M}_{\text {Earth }}$ with periods respectively between 0.7 and 2.4 days.

\section{References}

Boisse, I., Moutou, C., Vidal-Madjar, A., et al. 2009, A\&SA, 495, 959

Desort, M., Lagrange, A.-M., Galland, F., et al. 2007, A\&BA, 473, 983

Queloz, D., Henry, G. W., Sivan, J. P., et al. 2001, A\&A, 379, 279

Queloz, D., Bouchy, F., Moutou, C., Hatzes, A., Hébrard, G., et al. 2009, A\& $A$, 506, 303

Vauclair, S., Laymand, M., Bouchy, F., et al. 2008, A\&A, 428, 5 